\title{
Spilled Gallstone Abscesses Mimicking Liver Metastases
}

\author{
*Scott Matthews and Mark Taylor \\ Queens University Belfast, UK
}

Submission: August 18, 2016; Published: August 29, 2016

*Corresponding author: Scott Matthews, Queens University Belfast, 30 Holstein Crescent Lisburn Co. Antrim BT28 2RS, United Kingdom.

\begin{abstract}
We present a unique case highlighting the difficulty relating to the specificity of CT-PET following a case where gallstone abscess mimicked colorectal liver metastases. CT-PET is often used in the diagnostic work-up of patients with colorectal liver metastatic disease; aiding staging and patient management. However, care must be taken in the interpretation of PET scan hot spots and must be considered along with clinical history and the other imaging modalities used. This case highlights that the interpretation of imaging modalities can overcall the diagnosis in patients with presumed colorectal liver metastases.
\end{abstract}

Keywords: Spilled gallstone; Mimicking liver metastases; CT-PET; Colorectal liver metastases; Hot spots; Excision of Retro-caecal lesion

\section{Introduction}

We present a unique case highlighting the difficulty relating to the specificity of CT-PET following a case where gallstone abscess mimicked colorectal liver metastases. CT-PET is often used in the diagnostic work-up of patients with colorectal liver metastatic disease; aiding staging and patient management. However, care must be taken in the interpretation of 'hot spots' and must be considered along with clinical history and the other imaging modalities used. This case highlights that the interpretation of imaging modalities can overcall the diagnosis in patients with presumed colorectal liver metastases.

\section{Case Vignette}

Mrs G., a 63 year old female with a background of laparoscopic cholecystectomy (2015), asthma and hypercholesterolaemia was admitted electively to the Mater Hospital on 21st March 2016 for a right hepatectomy and excision of retro-caecal lesion. One year previously she underwent an anterior resection for a T2 NO MO rectal adenocarcinoma. A rise in CEA prompted an urgent interval CT abdomen and pelvis which identified a $4 \times 2 \mathrm{~cm}$ abnormality at the gallbladder apex. On CT PET in February 2016 multiple FDG avid liver lesions were reported as colorectal liver metastases and following MDM discussion she was boarded for a right hepatectomy. Intra operatively multiple gallstone deposits within the peritoneum and diaphragm were observed and it became apparent that the previous laparoscopic cholecystectomy was in fact a subtotal procedure with a significant proportion of the gallbladder remaining in situ. Spilled gallstones were identified as the cause of the deposits on the surface of the liver. Intraoperative ultrasonography revealed multiple collections of gallstones in areas similar to the FDG avid lesions on the CT-PET scan. These were all on the serosal surface of the liver (Figure 1).

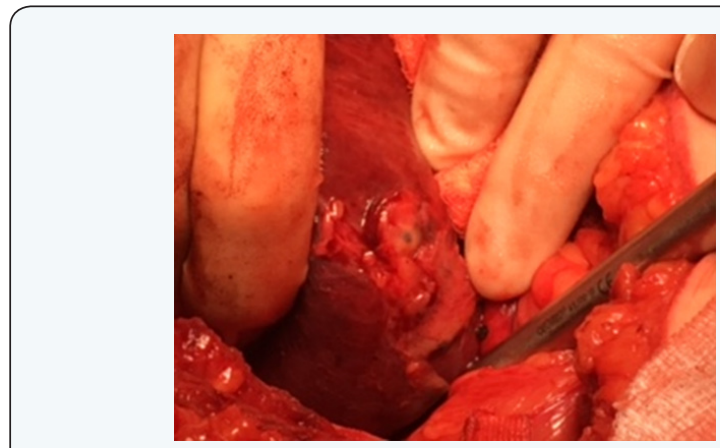

Figure 1: Gallstone Abscess within Liver Serosa.

There was no evidence of intrahepatic lesions in keeping with colorectal liver metastases. The only disease present was multiple gallstone abscesses adherent to serosal surface of liver. All visible gallstone abscesses were incised and drained. This is addition the retrocaecal lesion were sent to histopathology with multiple pus $\mathrm{O}+\mathrm{S}$ swabs sent also. A completion cholecystectomy was performed followed by a thorough saline washout to remove contamination and reduce infection risk (Figure 2). A decision was taken not to proceed with a right hepatectomy following a negative frozen section from one of the lesions. Postoperatively she was commenced on a three-day course of intravenous Tazocin. She recovered well postoperatively and was discharged home on day 5 following drain removals. Histopathology confirmed 
the specimens were gallstone abscesses with no evidence of malignancy or dysplasia.

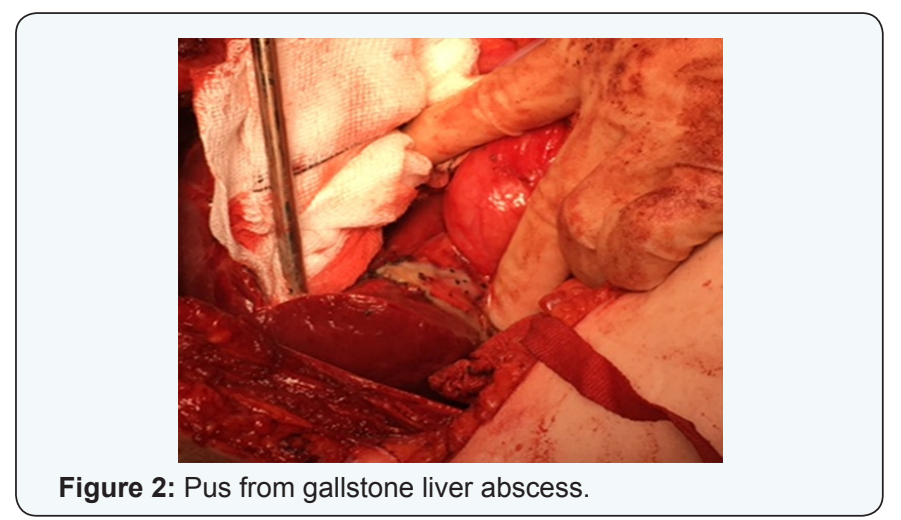

\section{Discussion}

This case highlights an important differential of surface 'hot spots' on the liver in a patient with a past history of laparoscopic cholecystectomy and colorectal cancer. The history of lesions following the resection of a primary cancer, in association with them being FDG-avid led to the conclusion that this lady had metastatic colorectal liver metastases. Sacks described a combined sensitivity of $88 \%$ and specificity of $96 \%$ for hepatic disease. Despite this high specificity this case highlights the importance of differential diagnoses [1]. However; this case demonstrates that benign gallstone abscesses can be misrepresented as metastatic cancer. Last year a similar case was documented where an enhancing liver lesion was confused on both MRI and PET as a potential liver malignancy but biopsies revealed no malignancy [2] (Figure 3).

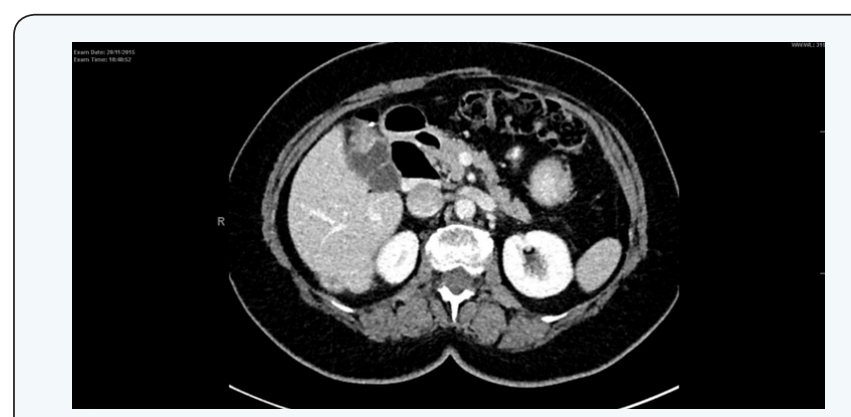

Figure 3: Abnormal area at gallbladder apex.

Despite a laparotomy this was a fantastic outcome for the patient as it proved with frozen section that she did not have colorectal liver metastases thus reducing morbidity and improving her survival rate. Three months postoperatively a follow-up CT scan revealed potential areas of concern with regards to metastatic disease. However, these are likely unobserved gallstone abscess that were spilling from the liver serosa. An MRI has been requested despite this to further rule out metastatic disease given the previous colonic cancer. The patient herself though is clinically well and has recovered well from the laparotomy with a return to work. The lesson from this case is that lost stones should be retrieved at the time of surgery [3-5]. 40\% of laparoscopic cholecystectomies the gold standard treatment of gallstones have a varying degree of stone spillage $[5,6]$. In most patients no complications occur [3].

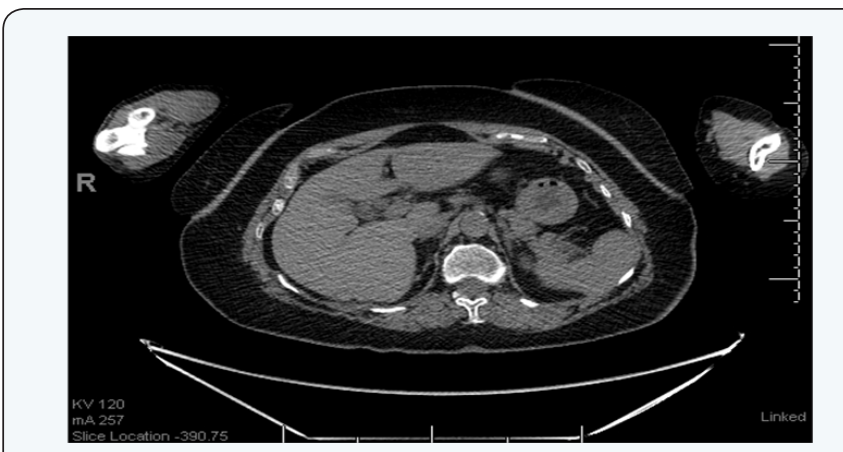

Figure 4: Abnormal area in liver serosa due to gallstone liver abscess.

The incidence of complications is low enough however that conversion to laparotomy is not justified for stones that are not retrievable laparoscopically [6]. Caution should therefore be exercised during dissection of the gallbladder to avoid perforating the gallbladder in the first instance. This involves both technical and non-technical skills on the surgeon and assistant's behalf (Figure 4). Considering the approximately 10\% incidence of serious inflammatory complications of lost stones, patients should be informed if this occurs [4]. It should also be documented in the operation note/discharge letter to allow for earlier diagnosis of potential complications later [4]. Complications can be compounded further if there is incidental gallbladder carcinoma (0.6-2.1\%) on histology as spilled tumour bedded stones could potentially cause disease recurrence if not removed [3]. It is therefore essential that any cholecystectomies whether total or subtotal with gallstone leakage is well documented and that this is clearly highlighted in the patients past surgical history under the named procedure [6].

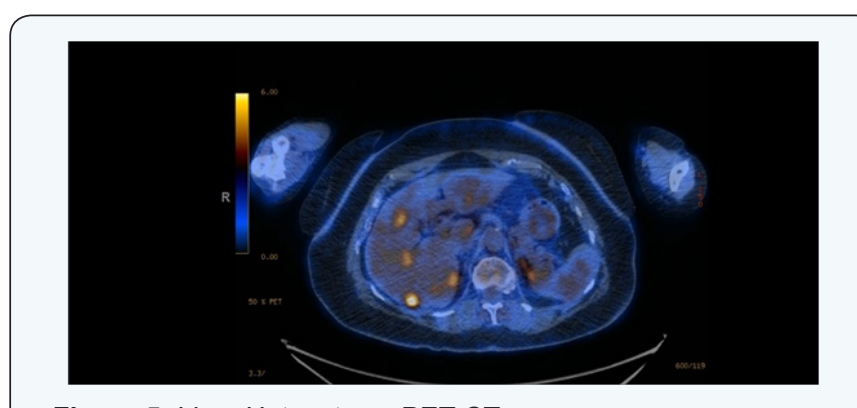

Figure 5: Liver Hotspots on PET-CT.

This ensures effective communication between colleagues so all are aware of the previous morbidity. Patients should be informed of the lost gallstones to enable them to alert medical professionals on history taking so potential complications can be considered and also allow radiologists to be aware of them on medical imaging (Figure 5). Complications can be extensive including mimicking peritoneal metastases, inflammation, fistula, abscess and peritonitis, fibrosis, adhesions [2,5-10]. However, the majority of patients remain asymptomatic [4]. 


\section{Conclusion}

This case demonstrated how spilled gallstones from a previous subtotal cholecystectomy led to gallstone abscesses on the liver serosa which mimicked colorectal liver metastases on preoperative imaging. This highlights the importance of remembering the differential diagnoses despite the high specificity of CT-PET. The use of frozen section should be commended in situations where there is diagnostic doubt at time of surgery which in this case altered the overall management plan. It is essential that care is taken to avoid spilled stones and all efforts are made to retrieve those spilled within laparoscopic limits. Thorough documentation of spilled stones as well as informing patients may help highlight spilled stones complications earlier and make the clinician consider this as a potential differential diagnosis.

\section{References}

1. Sacks A, Peller PJ, Surasi DS, Chatburn L, Mercier G, et al. (2011) Value of PET/CT in the management of liver Metastases, part 1. AJR Am J Roentgenol 197(2): W256-W259.

2. Karabulut N, Tavasli B, Kiroğlu Y (2008) Intra-abdominal spilled gallstones simulating peritoneal metastasis: CT and MR imaging features (2008: 1b). Eur Radiol 18(4): 851-854.
3. Ahmad J, Mayne AI, Zen Y, Loughrey MB, Kelly P, et al. (2014) Spilled gallstones during laparoscopic cholecystectomy. Ann R Coll Surg Engl 96(5): 18-20.

4. Lee W, Kwon J (2013) Fate of lost gallstones during laparoscopic cholecystectomy. Korean J Hepatobiliary Pancreat Surg 17(2): 66-69.

5. Memon MA, Jenkins HJ, Fitzgibbons RJ (1997) Spontaneous erosion of a lost intra-abdominal gallstone through the back eight months following laparoscopic cholecystectomy. JSLS 1(2): 153-157.

6. Dasari BV, Loan W, Carey DP (2009) Spilled gallstones mimicking peritoneal metastases. JSLS 13(1): 73-76.

7. Memon MA, Deeik RK, Maffi TR, Fitzgibbons RJ (1999) The outcome of unretrieved gallstones in the peritoneal cavity during laparoscopic cholecystectomy. A prospective analysis. Surg Endosc 13(9): 848-857.

8. Cholecystectomy. JSLS: Journal of the Society of Laparoendoscopic Surgeons/Society of Laparoendoscopic Surgeons 1(2): 153-157. Inline Citation:(Memon, Jenkins, and Fitzgibbons, 1997)

9. Zehetner J, Shamiyeh A, Wayand W (2007) Lost gallstones in laparoscopic cholecystecomy- all possible complications. Am J Surg 193(1): 73-78.

10. Kim J, Siegel A, Yen SP, Seltzer M (2015) Subdiaphragmatic gallstone mimicking hepatic malignancy on FDG PET/CT. Clin Nucl Med 40(4): 347-348. 